\title{
Microbiological Profile of Chronic Osteomyelitis in a Tertiary Care Hospital
}

\author{
B. Padmini ${ }^{1}$ and S. Deepa ${ }^{2 *}$ \\ ${ }^{1}$ Department of Microbiology, Coimbatore Medical College, Coimbatore, Tamil Nadu, India \\ ${ }^{2}$ Department of Microbiology, Govt. Mohan Kumaramangalam Medical College, Salem, \\ Tamil Nadu, India \\ *Corresponding author
}

Osteomyelitis is the infection and inflammation of the bone. Inappropriate use of antibiotics and multidrug resistance has raised the morbidity and mortality

Keywords

Poor blood supply, diabetic wounds, loss of protective sensation and altered immune defenses

\section{Article Info}

Accepted:

12 April 2021

Available Online:

10 May 2021 rate in chronic osteomyelitis. This study aims to determine the bacterial profile and antimicrobial susceptibility patterns of chronic osteomyelitis with special mention to various resistant mechanisms. Samples from osteomyelitis cases were aerobically cultured and isolates from culture positives were identified by standard procedures. Antimicrobial susceptibility testing was done by Kirby Bauer disk diffusion method. Staphylococcal isolates were screened for methicillin resistance. The aerobic bacteriological study of chronic osteomyelitis showed Staphylococcus aureus is being continued to be major etiological agent followed by Pseudomonas aeruginosa and CoNS. Grampositive isolates were sensitive to linezolid, Vancomycin while gram-negative isolates were sensitive to Meropenem and Pipericillin with Tazobactum in the majority. In present study prevalence of methicillin-resistant Staphylococci aureus was found to be on the higher side. Antibiotic therapy on the basis of antibiotic susceptibility pattern helps the clinician to choose appropriate drugs leading to successful treatment and prevention of emergence and dissemination of drug resistant isolates.

\section{Introduction}

Osteomyelitis is an inflammatory process of bone and bone marrow caused by infecting microorganisms which results in local bone destruction, necrosis and bone neoformation (3). Osteomyelitis can infect all bones especially long bones and attacks all ages. Many classification systems for osteomyelitis have been presented based on the following (16).

Duration of the disease - Acute Osteomyelitis or Chronic Osteomyelitis. 
Origin of the infection - Hematogenous spread, contiguous contamination and vascular or neurologic insufficiency associated infection.

Primary hematogenous spread is bone infection that has been seeded through the bloodstream of bacteria (2) Contiguous infection is usually spread from a contaminated site, most commonly seen with direct contamination of bacteria in open fractures or joint replacement surgery with an orthopedic implant (3). Vascular or neurologic insufficiency associated osteomyelitis results from poor blood supply, diabetic wounds, loss of protective sensation and altered immune defenses, commonly affecting the lower extremity (17).

Acute osteomyelitis can be further subdivided into suppurative and nonsuppurative forms as well as progressive or hematogenous forms. Chronic osteomyelitis may be classified by the causative agent as suppurative or nonsuppurative forms or sclerosing with sub classifications of diffuse or focal disease. Chronic osteomyelitis is a relapsing and persistent infection that evolves over months to years and is characterized by low-grade inflammation, presence of dead bone (sequestrum), new bone apposition, and fistulous tracts. The treatment requiring surgical interventions and long-term broad spectrum antibiotics Chronic osteomyelitis commonly involves long bones; especially tibia and femur. Chronic infections do occur in children, generally as a consequence of failed antimicrobial therapy or the presence of an orthopaedic implant $(16,17)$.

The microorganisms may gain access to the bones during stabilization of fracture or implanting prosthesis. Prosthetic implants create an environment which favours microbial colonization and establishment of infection successfully in the bone. The infective agents adhere to foreign material in the body and secrete glycocalyx (biofilm formation) that inhibits the host defence mechanism and action of antibiotics so that infection can be established which would be difficult to eradicate $(1,10)$. In adults, the sinus is not closed and the persistence of viable pathogens in cavities for a longer period leads to reactivation of infection at any time.

The infection is commonly caused by pyogenic bacteria or mycobacteria and rarely by fungus which occurs especially in immunocompromised patients.

A single pathogenic organism is almost always recovered from the bone in haematogenous osteomyelitis, whereas, multiple organisms are usually isolated in contiguous focus osteomyelitis, especially in the diabetic foot (18). The bacteria most commonly isolated from chronic osteomyelitis are Staphylococcus aureus, coagulase negative staphylococci (especially in implantassociated infections), Pseudomonas spp., Escherichia coli, Proteus spp., Klebsiella spp., Enterococcus spp., Enterobacter spp. and anaerobes like Peptostreptococcus spp., Bacteroides spp., Clostridium spp. and rarely Salmonella spp. (in individuals with sickle cell disease) and Actinomycetes $(1,7)$.

Osteomyelitis is an ongoing problem due to emergence of the pattern and behaviour of organisms are constantly changing under the pressure of newer antibiotics. With this background, the present study was conducted to study the spectrum of organisms causing chronic osteomyelitis and their antimicrobial susceptibility pattern for ensuring proper treatment of the patients.

\section{Materials and Methods}

This is a prospective observational study done at Microbiology Department in Coimbatore 
Medical College and hospital for a period of 1 year. A total number of 23 clinically diagnosed cases of chronic osteomyelitis were included in the study.

\section{Inclusion Criteria}

Patients admitted to orthopaedic wards and those attending outpatient departments who satisfy one of the following components of chronic osteomyelitis.

Presence of sinus/sequestrum or sclerosis /Intra operative pus.

Patients older than 12 years.

Radiological changes suggestive of infection for 6 weeks or more.

Even after treatment, persistence or relapse of infection.

\section{Exclusion Criteria}

Pediatric age group ( $<12$ years).

Patients who had shorter than 4-week history.

\section{Collection, transport and processing of samples}

Samples like pus, sinus discharge or exudates were collected. Two swabs for each specimen was collected aseptically. After the collection of specimen, they were immediately processed in microbiology laboratory according to the standard operative procedure $(11,12)$. The first swab was used for direct gram staining and was examined under oil immersion objective for the presence of inflammatory cells and bacteria while second swab was inoculated on Blood agar, Mac-conkey agar and nutrient agar(13). All plates were aerobically incubated overnight at $370 \mathrm{C}$. The isolated organisms were identified according to morphology in gram staining, colony characteristics and biochemical properties (13, 12). Antimicrobial susceptibility of bacterial isolates to the commonly used antibiotics was done by using modified Kirby Bauer disc diffusion method on Mueller Hinton agar equivalent to $0.5 \mathrm{McF}$ arland standard as per CLSI guidelines.

\section{Results and Discussion}

A total of 23 clinically diagnosed cases of osteomyelitis were included in the study of which $20(87 \%)$ were culture positive and $3(13 \%)$ had no growth in culture.

In this study, Out of 20 patients with Osteomyelitis, 19 were males and 1 was female.

In the present study, the predominantly affected age group was between 31-40 followed years by 21-30 yrs.

Of the 20 isolates $60 \%$ were gram positive cocci followed by Gram negative bacilli (40\%). Among the gram positive isolates Staphylococcus aureus (40\%) was the predominant organism followed by CoNS (20\%). Among the Gram negative bacilli maximum were Pseudomonas aeruginosa (30\%) followed by Proteus (10\%).

In the present study Staphylococcus aureus showed $100 \%$ sensitivity to Vancomycin and Linezolid, 60-75\% sensitivity to Erythromycin and Doxycycline.

The next common organism was CoNS with $100 \%$ sensitivity to Vancomycin and Linezolid. The sensitivity to Doxycycline was $75 \%$.

Out of 8 Staphylococcus aureus isolates 6 (75\%) were Methicillin resistant. Two out of 4 CoNS isolates (50\%) were Methicillin resistant. 
In this present study, Pseudomonas aeruginosa showed $100 \%$ sensitivity to Meropenem, Piperacillin tazobactam, Ceftazidime and Amikacin. The sensitivity to Gentamicin, Ciprofloxacin and Cefotaxime were $80 \%, 60 \%$ and $40 \%$ respectively. Proteus was $100 \%$ sensitive to Amikacin, Gentamicin, Ciprofloxacin, Meropenem, Piperacillin tazobactam and Ceftazidime. The sensitivity to Cefotaxime was $50 \%$

In present study, maximum osteomyelitis cases belonged to younger age groups of 3140 years $(60 \%)$ followed by $21-30$ years (25\%). This coincides with the study by Khatoon et al., (1). This may reflect the fact that the usage of motor vehicles is common among young adults and working community.

In the present study males were commonly affected when compared to females. This is in concordant with the study conducted by Devi et al., (2). This could be due to more frequent exposure of males to accidents, trauma and fracture.

Even though the gram negative organisms are increasing rapidly since longer time, still staphylococcus remained the most common isolate of osteomyelitis with methicillin resistant strains aggravating the disease further. Present study reported that Staphylococcus aureus was the commonest isolate from osteomyelitis cases (40\%), followed by Pseudomonas spp. (30\%), coagulase negative staphylococci (20\%) Proteus (10\%). This finding is in concordance with another study by Wadekar et al.,(5) Various previously done studies by Suguneswari et al., Rao et al., Zuluaga et al., and Ellies et al., $(6,7,9)$ also reported staphylococcus as the major isolate from osteomyelitis cases. Present study reported the prevalence of methicillin resistant staphylococci (MRSA) to be $66 \%$, with $75 \%$ among Staphylococcus aureus isolates and 50 $\%$ among coagulase negative staphylococci. All the MRSA isolates were $100 \%$ sensitive to vancomycin and linezolid followed by sensitivity to erythromycin \& Doxycycline (60-75\%). This is similar to a previous study done by Wadekar et al., and Kaur et al., $(5,8)$. But Raviprakash et al., study showed only $47 \%$ of MRSA (4).

In present study amongst the Gram negative organisms isolated from osteomyelitis cases, maximum were Pseudomonas spp. (30\%), followed by Proteus spp. (10\%) and both showed $100 \%$ sensitivity to Meropenem, Piperacillin tazobactam, Ceftazidime and Amikacin. The sensitivity to Gentamicin, Ciprofloxacin and Cefotaxime were 80\%, 60\% and $40 \%$ respectively for Pseudomonas spp. Proteus was 50\% sensitive to Cefotaxime.

Table.1 Age-wise distribution of Osteomyelitis

\begin{tabular}{|c|c|}
\hline Age & No. of cases \\
\hline$<20$ yrs & 1 \\
\hline $21-30$ yrs & 5 \\
\hline $31-40$ yrs & 12 \\
\hline $41-50$ yrs & 1 \\
\hline$>50$ yrs & 1 \\
\hline
\end{tabular}


Table.2 Bacterial Profile of Osteomyelitis

\begin{tabular}{|c|c|}
\hline Name of the isolate & No. of isolates $(\mathbf{n}=\mathbf{2 0})$ \\
\hline S.aureus & $\mathbf{8}$ \\
\hline P.aeruginosa & 6 \\
\hline CoNS & 4 \\
\hline Proteus & 2 \\
\hline
\end{tabular}

Table.3 Antibiotic sensitivity pattern of gram positive organisms

\begin{tabular}{|c|c|c|c|c|c|c|c|c|c|}
\hline \multicolumn{8}{|c|}{ Antibiotic sensitivity tests of gram positive organisms } \\
\hline \multirow{2}{*}{ Microorganism } & Antibiotic sensitivity in percentage & (sensitive no./total tested no.) \\
\cline { 2 - 11 } & $\mathrm{P}$ & $\mathrm{G}$ & $\mathrm{CIP}$ & $\mathrm{COT}$ & $\mathrm{E}$ & $\mathrm{DO}$ & $\mathrm{CX}$ & Van & LZ \\
\hline Staphylococcus & - & $50 \%$ & $25 \%$ & $38 \%$ & $75 \%$ & $63 \%$ & $25 \%$ & $100 \%$ & $\mathbf{1 0 0 \%}$ \\
\hline aureus & $0 / 8$ & $4 / 8$ & $2 / 8$ & $3 / 8$ & $6 / 8$ & $5 / 8$ & $2 / 8$ & $8 / 8$ & $\mathbf{8 / 8}$ \\
\hline CoNS & - & $50 \%$ & $50 \%$ & $25 \%$ & $50 \%$ & $75 \%$ & $50 \%$ & $100 \%$ & $\mathbf{1 0 0 \%}$ \\
\hline & $\mathbf{0 / 4}$ & $\mathbf{2 / 4}$ & $\mathbf{2 / 4}$ & $\mathbf{1 / 4}$ & $\mathbf{2 / 4}$ & $\mathbf{3 / 4}$ & $\mathbf{2 / 4}$ & $\mathbf{4 / 4}$ & $\mathbf{4 / 4}$ \\
\hline
\end{tabular}

$\mathrm{P}$ - Pencillin, G-Gentamicin. CIP-Ciprofloxacin, COT-Cotrimoxazole, E-Erythromycin, DO-Doxycycline, CxCefoxitin, Van-Vancomycin, LZ-Linzolid.

Table.4 Antibiotic sensitivity pattern of gram negative organisms

\begin{tabular}{|c|c|c|c|c|c|c|c|c|c|}
\hline \multirow{2}{*}{ Microorganism } & \multicolumn{6}{|c|}{ Antibiotic sensitivity in percentage (sensitive no./total tested no.) } \\
\cline { 2 - 9 } & CIP & COT & AK & G & CT X & MRP & PIT & CAZ \\
\hline Pseudomonas & $50 \%$ & - & $100 \%$ & $83 \%$ & $50 \%$ & $100 \%$ & $100 \%$ & $\mathbf{1 0 0 \%}$ \\
\hline $\begin{array}{c}\text { aeruginosa } \\
\text { Proteus }\end{array}$ & $3 / 6$ & - & $6 / 6$ & $5 / 6$ & $3 / 6$ & $6 / 6$ & $6 / 6$ & $\mathbf{6 / 6}$ \\
\hline & $100 \%$ & $0 \%$ & $100 \%$ & $100 \%$ & $50 \%$ & $100 \%$ & $100 \%$ & $\mathbf{1 0 0 \%}$ \\
\hline & $\mathbf{2 / 2}$ & $\mathbf{0 / 2}$ & $\mathbf{2 / 2}$ & $\mathbf{2 / 2}$ & $\mathbf{1 / 2}$ & $\mathbf{2 / 2}$ & $\mathbf{2 / 2}$ & $\mathbf{2 / 2}$ \\
\hline
\end{tabular}

CIP-Ciprofloxacin, COT-Cotrimoxazole, Ak-Amikacin, CTX=Cefataxime, MRP-Meropenam, PITPiperacillintazobactum, CAZ-Ceftazidime, G-Gentamicin.

Fig.1 Outcome of Culture

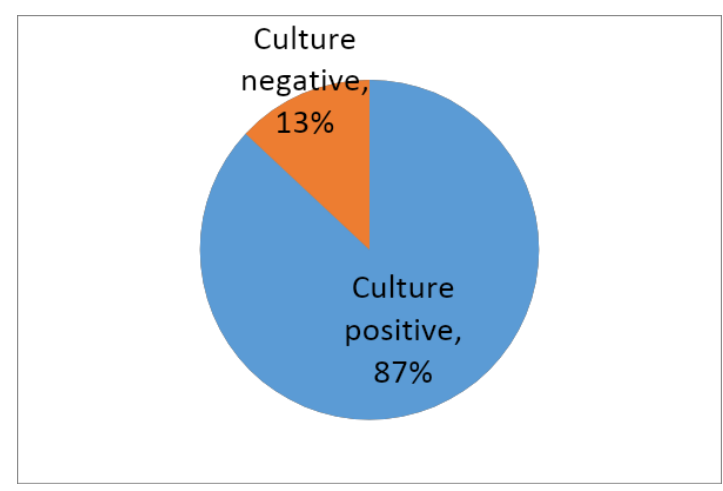


Fig.2 Gender-wise distribution of Osteomyelitis

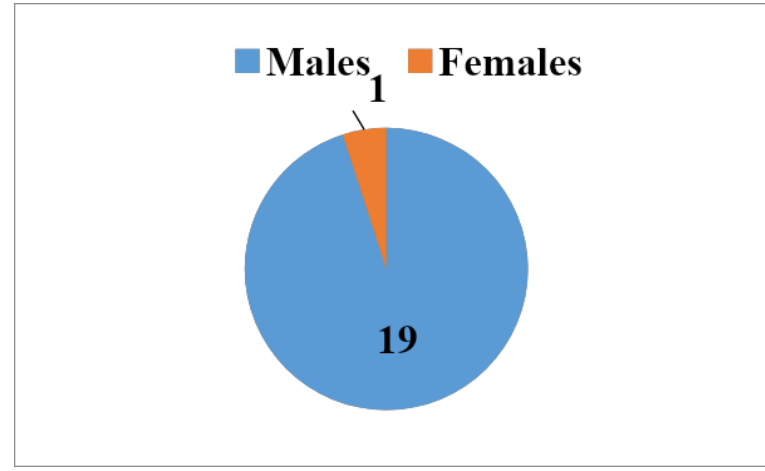

Fig.3 Age-wise distribution of Osteomyelitis

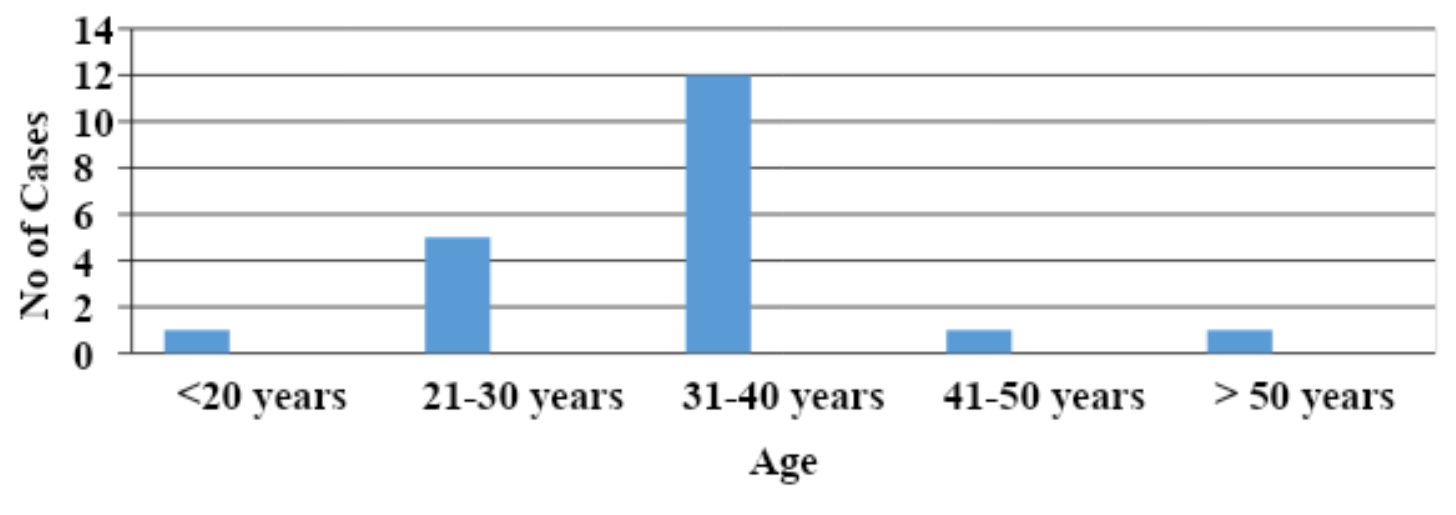

Fig.4 Bacterial Profile of Osteomyelitis

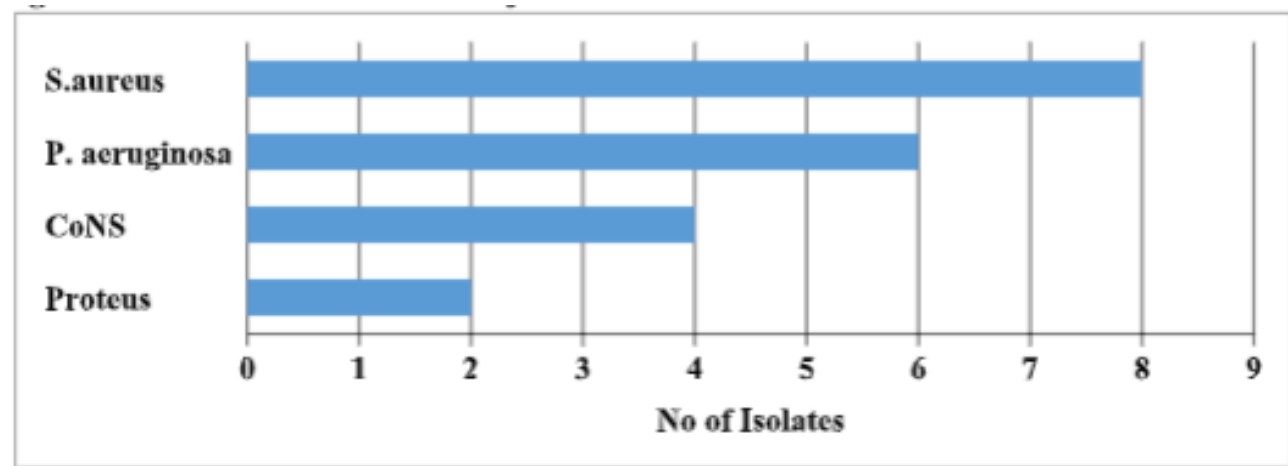


Fig.5 Antibiotic sensitivity pattern of gram positive organisms

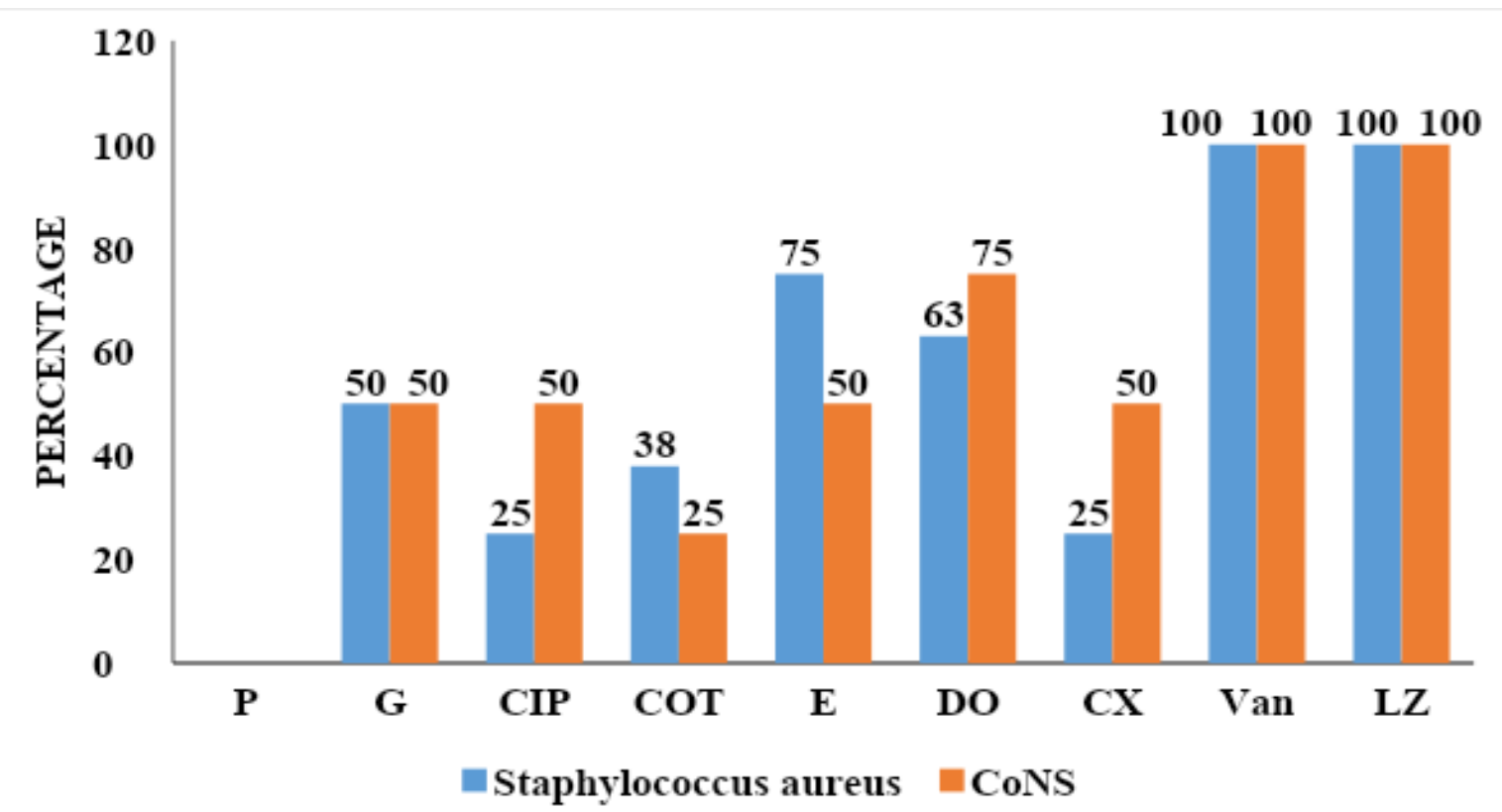

*** P - Pencillin, G-Gentamicin. CIP-Ciprofloxacin, COT-Cotrimoxazole, E-Erythromycin, DO-Doxycycline, CXCefoxitin, Van-Vancomycin, LZ-Linzolid.

Fig.6 Antibiotic sensitivity pattern of gram negative organisms

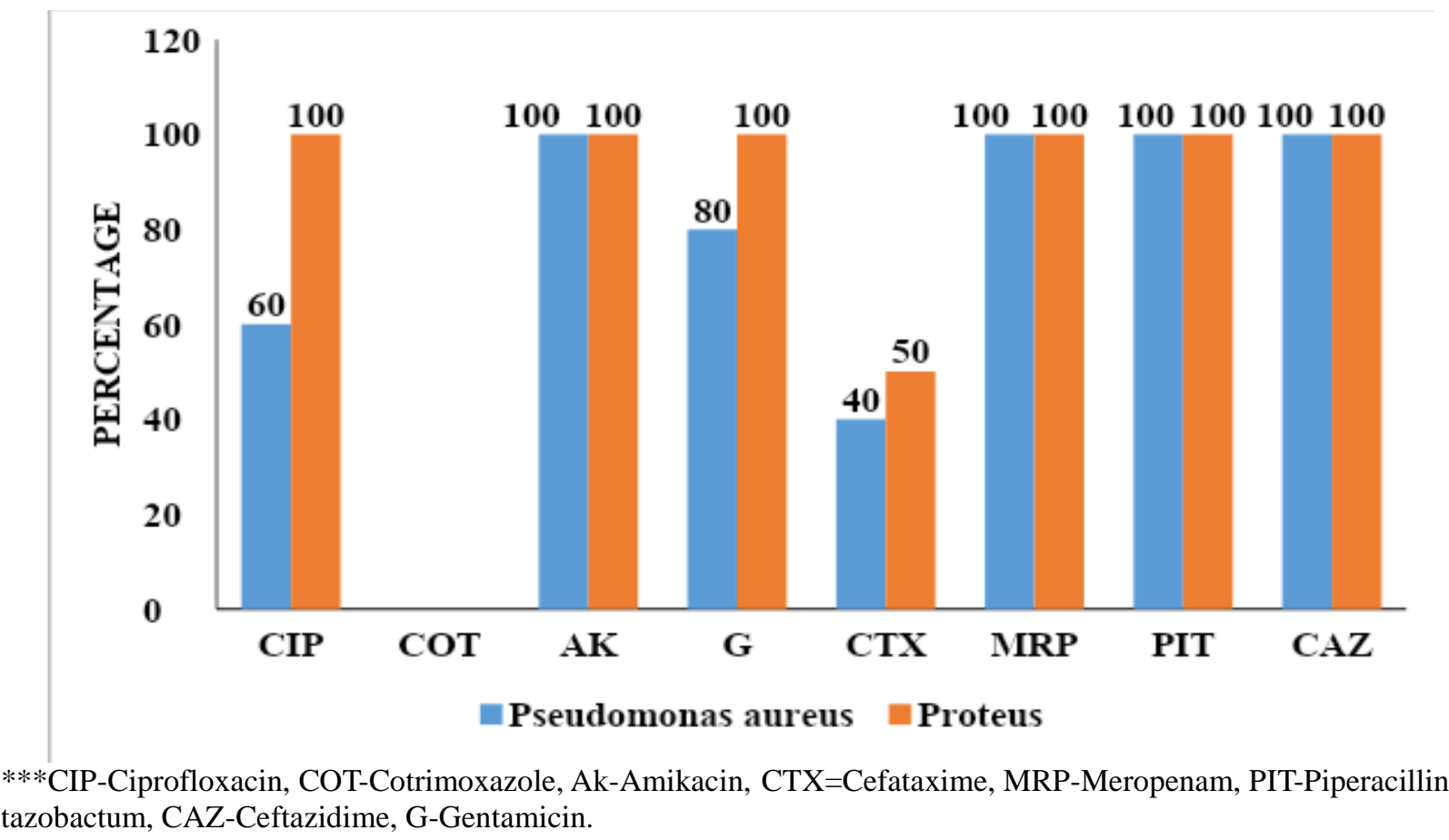

This is in accordance with a study by Suguneswari et al., (9). But this is in contrast to study by Wirbel et al., study. In this study, bacterial pathogens responsible for infection 
were mainly monomicrobial which is in accordance with the study Qurahi et al., Osteomyelitis has been the major cause of morbidity since long.

Emerging multidrug resistant strains is of major concern as they pose challenge in the treatment of osteomyelitis. In present study the methicillin resistant staphylococci is found to be quite high among the organisms isolated from osteomyelitis patients.

MRSA is usually spread by direct contact with an infected wound or by contaminated items like bandages or from contaminated hands usually those of health providers resulting in life threatening infections like bloodstream infections, pneumonia, surgical site infection and sepsis. Healthcare facilities can make prevention of MRSA infections a priority, assess their relevant data, implement prevention actions like mainly contact precautions, hand hygiene with screening for colonisation of MRSA in nostrils, throat, and any skin lesions or wounds and evaluate the progress. The early detection of such drug resistant isolates may help in appropriate antimicrobial therapy since beginning and thus avoid the development and dissemination of these multidrug resistance strains in the hospital as well as in the community.

The present antibiotic resistance pattern in osteomyelitis with MRSA strains is alarming and calls for updating the antibiotic therapy guidelines in the country. Although, treatment of osteomyelitis is challenging, good results can be achieved by a single stage protocol including radical debridement in conjunction with systemic and topical antibiotic.

\section{References}

1. Khatoon. R. 2017. Antibiotic resistance pattern among aerobic bacterial isolates from osteomyelitis cases attending a
Tertiary care hospital of North India with special reference to ESBL, AmpC, MBL and MRSA production. Int J Res Med Sci. 5(2):482-490.

2. Devi. C, Banu S. T. A study on bacterial and fungal isolates and their antimicrobial susceptibility pattern in patients with chronic osteomyelitis in a tertiary care hospital. Innov Pharm Pharmacother. 2017;5(4):195-203.

3. Kuzma. J, Hombhanje.F. Chronic Osteomyelitis - Bacterial Flora, Antibiotic Sensitivity and Treatment Challenges. The Open Orthopaedics Journal. 2018; 12, 153163.

4. Raviprakash. V, Hanumanthapa. A. R, Viswanath. G. Prevalence of methicillin resistant Staphylococcus aureus in osteomyelitis. JMM Medical College, Davangere, Karnataka: Abstract Book XXVIII National Congress of Indian Association of Medical Microbiologist; 2004; BP-91, 89.

5. Wadekar. M. D, Naganath. M, Venkatesha. D. Detection of ESBL, MBL and MRSA among isolates of chronic osteomyelitis and their antibiogram. Int J Curr Microbiol App Sci. 2015; 4:289-95.

6. Rao. P. S, Beena. V. K, Shivnanda. P. G. Bacteriological study of bone and joint infections with special reference to anaerobes. Indian J Orthopaedics. 1997;31:171-4.

7. Zuluaga. A. F, Galvis. W, Jaimes. F, Vesga. O. Lack of microbiological concordance between bone and non-bone specimens in chronic osteomyelitis: an observational study. BMC Infect Dis. 2002;2:1-7.

8. Kaur. J, Gulati.V. L, Aggarwal. A, Gupta. V. Bacteriological profile of osteomyelitis with special reference to Staphylococcus aureus. Indian Journal for the Practising Doctor. 2008;4:6.

9. Suguneswari. G, Singh. A. H, Basu. R. Bacteriological profile of osteomyelitis in a 
tertiary care hospital at Visakhapatnam, Andhra Pradesh. Int J Cur Res Rev. 2013;5:52-8.

10. Waldvogel. F. A, Medoff. G, Swartz. M. N. Osteomyelitis - a review of clinical features, therapeutic considerations and unusual aspects. 3: osteomyelitis associated with vascular insufficiency. N Engl J Med. 1970;282:316-22.

11. Spellberg. B, Lipsky. B. A. Systemic antibiotic therapy for chronic osteomyelitis in adults. Clin Infect Dis 2012; 54(3): 393407.

12. Brady. R. A, Leid. J. G, Calhoun. J. H, Costerton. J, Shirtliff. M. E. Osteomyelitis and the role of biofilms in chronic infection.FEMS Immunol Med Microbiol.2008; 52(1): 13-22.

13. Institute. CaLS. Performance standards for antimicrobial susceptibility testing: Nineteenth informational supplement In: Institute CalS, editor. CLSI Document M100-S19. Wayne, Pennsylvania: Pennsylvania Clinical and laboratory Standards Institute; 2009.

14. Carvalho. V. C, Oliveira. P. R, Dal-Paz. K, Paula. A. P, Félix CdaS, Lima. A. L. Gram- negative osteomyelitis: Clinical and microbiological profile. Braz J Infect Dis. 2012;16(1): 63-7.

15. Kini. A. R, Shetty. V, Kumar. A. M, Shetty. S. M, Shetty. A. Communityassociated, methicillin-susceptible, and methicillin-resistant Staphylococcus aureus bone and joint infections in children: Experience from India. J Pediatr Orthop B. 2013; 22(2): 158-66.

16. Souvik Chakraborty, D. V. Gowda and Vishal N. gupta The Current Trend In Treatment For Osteomyelitis. International Journal of Pharmaceutical Sciences And Research

17. Mitchell C. Birt, David W. Anderson, E. Bruce Toby, and Jinxi Wang $\square$.J Orthop. 2017 Mar; 14(1): 45-52

18. Aparna Pandey, Prachi ShawlandAamir Johar. Bacteriological Profile of Chronic Osteomyelitis with Special Reference to Antibiotic Resistance Mechanisms/Patterns-A Cross-sectional Prospective Study from Tertiary Care Hospital in Central India. Journal of Advances in Medicine and Medical Research32(3): 43-52, 2020.

\section{How to cite this article:}

Padmini, B. and Deepa, S. 2021. Microbiological Profile of Chronic Osteomyelitis in A Tertiary Care Hospital. Int.J.Curr.Microbiol.App.Sci. 10(05): 826-834. doi: https://doi.org/10.20546/ijcmas.2021.1005.092 